\title{
Performance of Single Stage Four Nozzles Pintle Type Hydraulic Servovalves*
}

\author{
Mohamed A. ELGAMIL**, Saad A. KASSEM*** \\ and Hiroshi KANKI**
}

\begin{abstract}
In this paper, the static characteristics of the four-nozzle pintle type servovalve are studied theoretically, under constant supply pressure and constant supply flow conditions. The study is on an eye to the effect of the valve improper configuration, the required pintle driving torque, and the effect of using unsymmetrical loads on the static characteristics. Errors in valve control gaps resulting from inaccurate initial adjustment, wear or else showed to affect slightly the load flow at each load pressure and input displacement. Units driven by unsymmetrical actuators showed to have lower valve flow forces and higher valve efficiency than symmetrical actuators. The minimum flow gain and flow pressure coefficient are obtained when the load is symmetric. The study showed that the flow forces have two instability sources, decentering nature and similar values at different input displacements. The study discussed the selection of the pintle centering spring to overcome the problems of instability.
\end{abstract}

Key Words: Four Nozzle, Servovalves, Static Characteristics, Improper Configuration, Flow Force, Flow Torque, Pintle Instability, Unsymmetrical Loads, Valve Efficiency.

\section{Introduction}

Two nozzle-flapper servovalves are widely used as pilot stage in the two stage hydraulic servovalves, the main stage being usually of the spool type. The relatively large required driving torque with respect to the resulting hydraulic output power limits the use of two nozzle-flapper servovalves in higher output power applications. Four nozzle-flapper hydraulic servovalve, suggested by Williams ${ }^{(1)}$, has the remarkable merit of compensated hydrostatic forces on the bifurcated flapper, which keep the required driving force or torque within reasonable limits. The valve also has the merit of good linear static characteristics. Kassem and $\mathrm{Arafa}^{(2)}$ showed that for the original

* Received April 6th, 1998

** Graduate School of Science and Technology, Kobe University, Rokkodai 1-1, Nada-ku, Kobe 657-8501 Japan. E-mail : kanki@mech.kobe-u.ac.jp

*** Department of Mechanical Design and Production Engineering, Faculty of Engineering, Cairo University, Giza, Egypt valve (Fig. 1( a)), the resulting flow force on the flappers produce bending moment on the arms, a torque on the flapper cross piece, and a twist moment on its suspension. This leads to distort the postulated equality of the gapes and destroy the flapper or its parts when the valve output increases. They proposed to replace the bifurcated flapper with a pintle as shown in Fig. 1(b). In this paper, a four-nozzle servovalve, which replaces the flapper by a pintle is

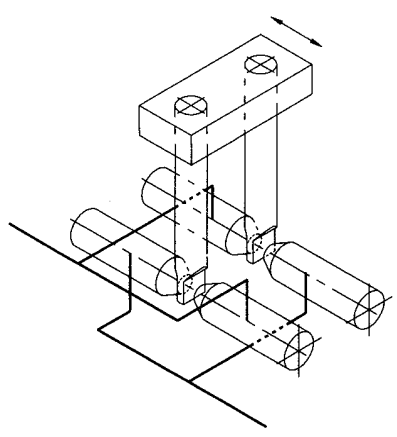

(a) original

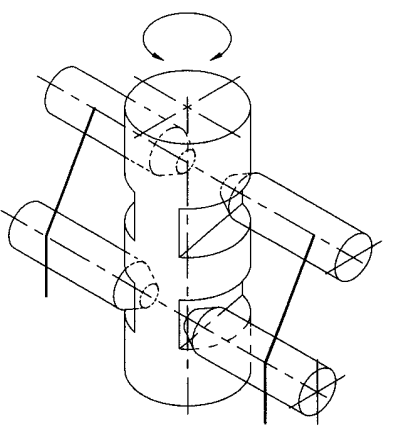

(b) after (2)
Fig. 1 Four-nozzle servovalve 
studied on an eye to the effect of the valve improper configuration, the required pintle driving torque, and the effect of using unsymmetrical actuators on the static characteristics.

\section{Valve Mathematical Model}

Figure 1 (b) shows the four-nozzle servovalve layout after Kassem and $\mathrm{Arafa}^{(2)}$, which is considered in this paper. The nozzles are arranged in two levels in front of flattened parts of the pintle. The flattened parts are chambers separated by the pintle shanks. One of the chambers is connected with the power supply, while the other is connected with the return line. Each two nozzles are connected with one of the two load lines. The two nozzles are located at different chambers and in opposite sides around the pintle center in such a way that, turning the pintle increases the gap in front of one of them and reduces the gap in front of the other. Figure 2 shows a schematic layout of a valve with an improper pintle, where all nozzles and chambers are drawn in one level for presentation simplicity. The assumed errors in the pintle configuration may present different defects in the valve, such as when a nozzle is not correctly located away from the pintle, or when the null gap area between a nozzle and the pintle changes due to wear or else. Figure 2 also shows the valve pintle when given an angular displacement $\theta$ to drive an unsymmetrical actuator. The control gap area in

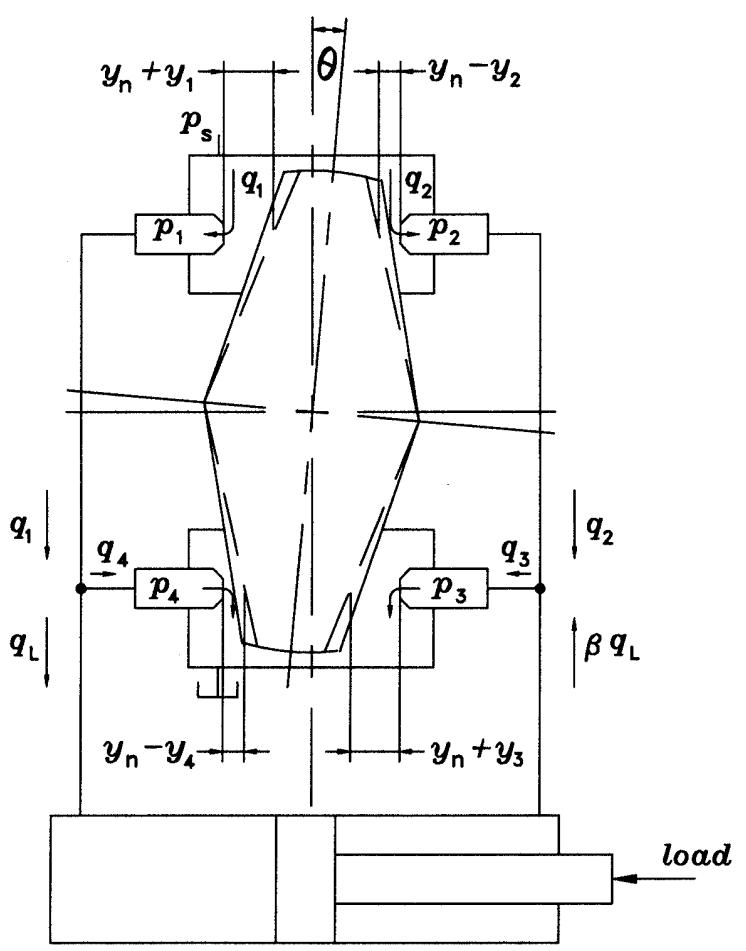

Fig. 2 Unsymmetrical load driven by a valve with improper configuration front of each nozzle can be expressed in terms of the linear distance $y$ measured at the nozzle center line, since for small value of angular input $\theta$, the variation of $y$ with $\theta$ was shown by Bahr ${ }^{(5)}$ to be linear. In other words, $(\theta=C y)$ where $C$ is constant. Referring to Fig. 2, the following equations governing the valve static characteristics were shown by Elgamil ${ }^{(3)}$ to be as follows ;

$$
\begin{aligned}
& q_{1}+q_{2}=q_{s} \\
& q_{1}-q_{4}=q_{L} \\
& q_{3}-q_{2}=\beta . q_{L} \\
& p_{1}=p_{4} \\
& p_{2}=p_{3} \\
& p_{L}=p_{1}-\beta . p_{2}
\end{aligned}
$$

where $p$ is pressure, $q$ is flow rate, the numerical subscript represents the nozzle number, and the subscripts $s$ and $L$ represent the supply and the load respectively. Referring to Fig. $2, y_{i}=y-j^{2 i} e_{i}, i=1,2$, 3 and 4 where $y$ is the input displacement, $y_{i}$ is the gap length facing the nozzle number, $i, e_{i}$ (the linear distance in front of the nozzle between the proper pintle face (the continuous line) and the defected surface (the dashed line)) is the error in this gap, $i=$ $\sqrt{-1}$, and $\beta$ is the actuator area ratio. Defining the term, $b=C_{d} \pi d_{n} y_{n} \sqrt{2 / \rho}$, where $C_{d}$ is the discharge coefficient, $d_{n}$ is the nozzle diameter, $y_{n}$ is the null gap length and $\rho$ is the flow density, the equations of flow rates through the nozzles may be written as follows;

$$
\begin{aligned}
& q_{1}=b\left(1+Y_{1}\right) \sqrt{\left|\left(p_{s}-p_{1}\right)\right|} \cdot \operatorname{sign}\left(p_{s}-p_{1}\right) \\
& q_{2}=b\left(1-Y_{2}\right) \sqrt{\left|\left(p_{s}-p_{2}\right)\right|} \cdot \operatorname{sign}\left(p_{s}-p_{2}\right) \\
& q_{3}=b\left(1+Y_{3}\right) \sqrt{\left|p_{3}\right|} \cdot \operatorname{sign}\left(p_{3}\right) \\
& q_{4}=b\left(1-Y_{4}\right) \sqrt{\left|p_{4}\right|} \cdot \operatorname{sign}\left(p_{4}\right)
\end{aligned}
$$

where, $Y_{i}=y_{i} / y_{n}, i=1,2,3$ and 4 .

The valve output power $p o$ and the valve efficiency $\eta$ can be calculated as follows

$$
\begin{gathered}
p o=p_{L} q_{L} \\
\eta=\frac{p_{L} q_{L}}{p_{s} q_{s}}
\end{gathered}
$$

Referring to Fig. 3, the flow forces acting on the pintle were derived by Kassem and $\mathrm{Arafa}^{(2)}$ and Osman ${ }^{(6)}$ and are given as ;

$$
\begin{aligned}
& F_{1}=-A p_{1}-\frac{0.5 \rho q_{1}^{2}}{A} \\
& F_{2}=-A p_{2}-\frac{0.5 \rho q_{2}^{2}}{A} \\
& F_{3}=A p_{3}+\frac{0.5 \rho q_{3}^{2}}{A} \\
& F_{4}=A p_{4}+\frac{0.5 \rho q_{4}^{2}}{A}
\end{aligned}
$$

where the nozzle cross sectional area $A=0.25 \pi d_{n}^{2}$. The force in each equation is obtained from the static pressure force at the nozzle face (the static pressure inside the nozzle minus the dynamic pressure) plus the flow momentum force due to changing its direction. 
The flow torque $m_{f}$ in the direction shown in Fig. 3 , is thus given by

$$
m_{f}=B\left(F_{2}+F_{3}-F_{1}-F_{4}\right)
$$

where $B$ is the perpendicular distance between the pintle center and the forces' lines of action (nozzles' centerlines). Substituting from Eqs. (13) to (16) into Eq. (17) the flow torque may be written as;

$$
m_{f}=\frac{0.5 \rho B}{A}\left(q_{1}^{2}+q_{3}^{2}-q_{2}^{2}-q_{4}^{2}\right)
$$

If $m_{f}$ as calculated from the foregoing equation is positive, then it is of a decentering nature, and the need arises for using a centering spring, e.g. a torsion bar. In this case the total torque $m$ required to be overcome by the driving torque is

$$
m=\frac{0.5 \rho B}{A}\left(q_{1}^{2}+q_{3}^{2}-q_{2}^{2}-q_{4}^{2}\right)-k \theta
$$

where $k$ is the torsional stiffness of the centering spring.

The foregoing equations are valid only when all the line pressures are higher than zero, otherwise cavitation would develop.

Generally, the valve is operated under constant supply pressure or constant supply flow. The valve static characteristics under these two supply conditions will be dealt with in the following.

\section{Valve Static Characteristics under Constant Supply Pressure Conditions}

In this case the supply pressure is constant. The

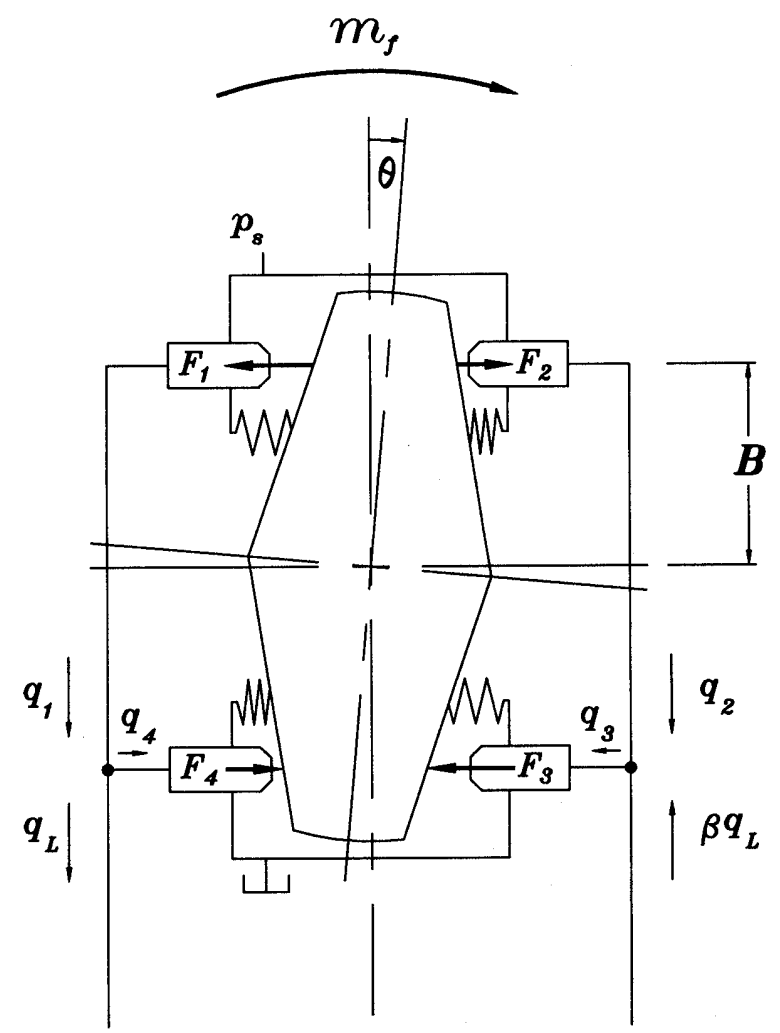

Fig. 3 Flow forces associated with flow through nozzle governing equations can be reduced to dimensionless forms using the following reference quantities ;

$i$-the reference pressure is the supply pressure $p_{r}=p_{s}$

$i i$-the reference flow is the valve null supply flow $q_{r}=b \sqrt{2 p_{r}}$

$i i i$-the reference input displacement is the null gap $y_{r}=y_{n}$

$i v$-the reference torque is taken as ;

$$
m_{r}=\frac{\rho . B}{2 A} q_{r}^{2}
$$

$v$-the dimensionless stiffness is taken as

$$
K=\frac{2 k \cdot C \cdot A \cdot y_{n}}{\rho \cdot B \cdot q_{r}^{2}}
$$

Uppercase letters are used to express the quantities in dimensionless forms. Using the reference values, the required driving torque in Eq. (19) may be written in dimensionless form as ;

$$
M=\left(Q_{1}^{2}+Q_{3}^{2}-Q_{2}^{2}-Q_{4}^{2}\right)-K \theta
$$

To avoid the cavitation the following limits can be derived;

a. For $P_{1}=P_{4}=0$

$$
Q_{L}=\frac{\left(1+Y_{1}\right)}{\sqrt{2}}
$$

b. For $P_{2}=P_{3}=0$

$$
Q_{L}=\frac{\left(1-Y_{2}\right)}{\sqrt{2} \beta}
$$

Figure 4 shows the variation of the dimensionless load pressure with the dimensionless load flow for various input displacement when $\beta=1$. It can be noticed that when $P_{L}$ is in the range between $(-0.5$ and +0.5 ), it is linearly dependent on the load flow $Q_{L}$ and the input displacement $Y$. Besides, it shows the case when there is positive or negative error of value $0.1 y_{n}$ that might occur in one side only. Case no. 1 occurs when $E_{1}$ or $E_{3}=0.1$ or when $E_{2}$ or $E_{4}=-0.1$ while case no. 2 occurs when $E_{1}$ or $E_{3}=-0.1$ or when

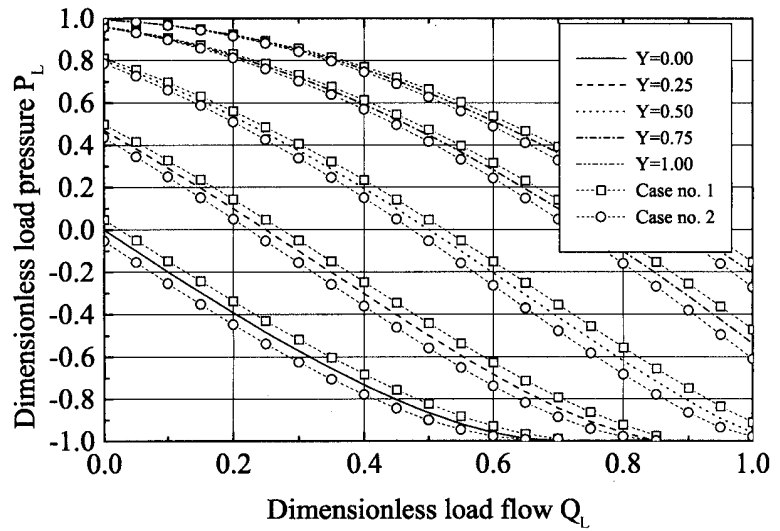

Fig. 4 Variation of dimensionless load pressure with dimensionless load flow for various dimensionless input displacement under constant supply pressure operation and errors effect when $\beta=1$ 
$E_{2}$ or $E_{4}=0.1$. The figure shows that the difference in the load flow is only dependent on the load pressure and not the input displacement and that they have nearly the same inclination at $P_{L}=0$ which means that the system damping ratio is kept nearly unchanged. The load flow difference decreases with load pressure increase. Figure 5 shows that the maximum supply flow is within 1.3 times the null flow rate.

To facilitate the comparison, Fig. 6 and Fig. 7 were drawn for both cases when $\beta=1$ and $\beta=0.5$ together. Figure 6 shows the variation of the dimensionless load pressure with the dimensionless load flow for various input displacements. The flow pressure coefficient $\left(-\partial Q_{L} / \partial P_{L}\right)$, which is related to the damping ratio (Merritt ${ }^{(7)}$ ), is bigger when $\beta=0.5$ than the flow pressure coefficient when $\beta=1$. Therefore, the minimum damping ratio is obtained when $\beta=1$. The flow gain $\left(\partial Q_{L} / \partial Y\right)$ is seen to increase when $\beta=0.5$, and consequently, both of the system accuracy and stability are influenced by the actuator area ratio selection. Cavitation is seen to develop in some zones in the second driven quadrant when $\beta=0.5$. Figure 7 shows the variation of the dimensionless flow torque with the dimensionless load flow for various input displacements, when the dimensionless stiffness $K=0$. The figure shows that the torque acting on the pintle due to the flow forces is of a decentering nature since the torque is positive in the first driving quadrant, i.e. in the same direction as the input displacement. This may cause valve instability since the flow torque tends to increase the input. It was shown by Elgamil ${ }^{(3)}$ that in the first driving quadrant, which is the usual working quadrant, there are intersections between the curves of different input displacements at the same torque. This means that for a certain driving torque, which is to be selected to compensate the flow decentering torque, the pintle can assume two

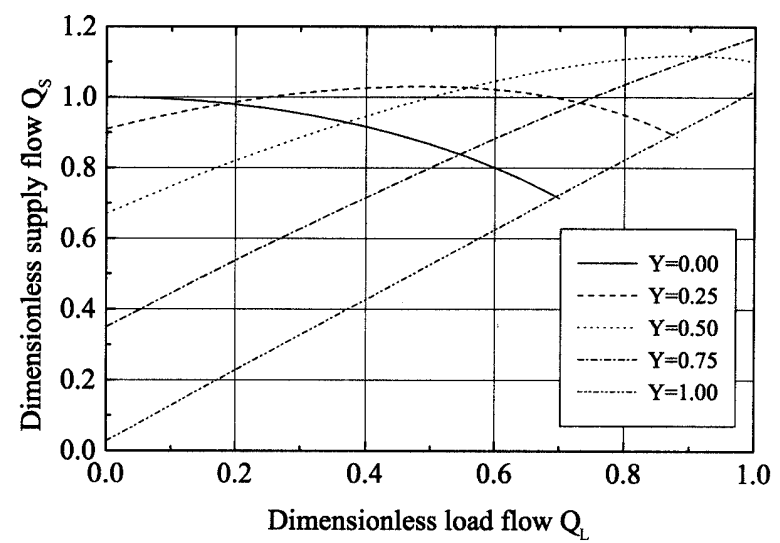

Fig. 5 Variation of dimensionless supply flow with dimensionless load flow for various dimensionless input displacement under constant supply pressure operation when $\beta=1$ different positions at the same load flow. This is another source of instability because the pintle will reciprocate between the two positions. Adding a centering spring would shift the curves downwards. Proper selection of stiffness may avoid the curves intersection because each input displacement curve shift is proportional to the input displacement value. Consequently, the initially intersected curves will be displaced from each other. Thus, the resultant torque due to both of the flow forces and the centering spring would be of a centering nature, where each torque value represents only one pintle position. Higher dimensionless stiffness results in larger curves shift. However higher dimensionless stiffness requires larger spring stiffness and required driving torque or less reference flow and consequently less supply pressure and output power. Equation (20) may be used to evaluate the maximum supply pressure, which insures operating a certain valve within a stable range.

In Fig. 6 it is shown that when $\beta=0.5$, the $P_{L}-Q_{L}$

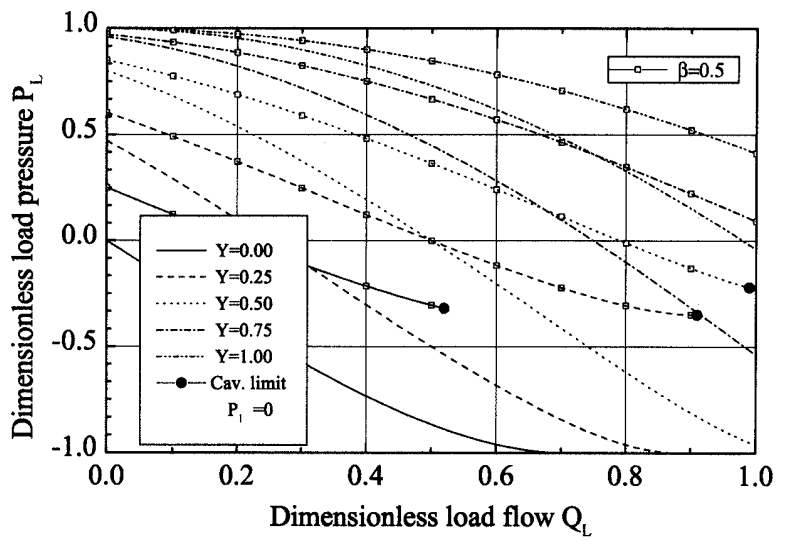

Fig. 6 Variation of dimensionless load pressure with dimensionless load flow for various dimensionless input displacement under constant supply pressure operation when $\beta=1$ and $\beta=0.5$

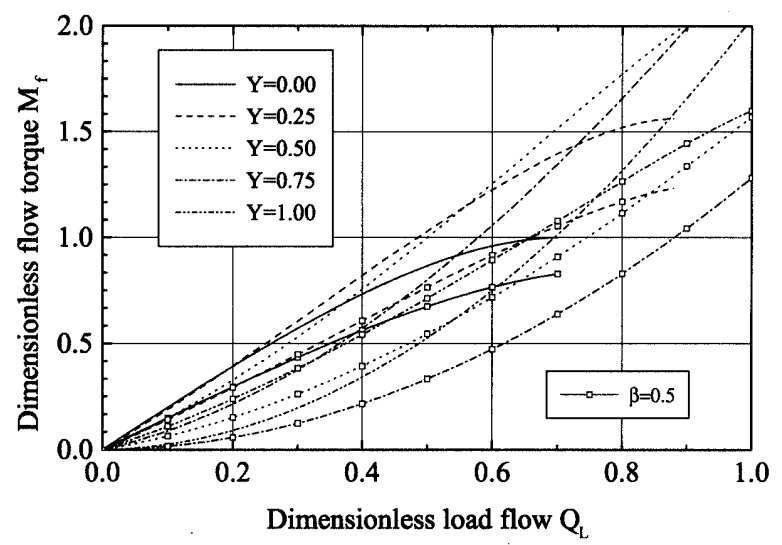

Fig. 7 Variation of dimensionless flow torque with dimensionless load flow for various dimensionless input displacement under constant supply pressure operation when $\beta=1$ and $\beta=0.5$ 
curve doesn't pass through the origin at zero input displacement. It means that the actuator may move even if there is no input signal. Referring to Fig. 7, since the flow torque is zero at $Q_{L}=0$ for all input displacements, the valve pintle null position may be set at the position which would keep the actuator stationary when $P_{L}=0$ instead of the mid way between the nozzles.

Figure 7 shows that the flow torque resulting when $\beta=0.5$ is less than that resulting when $\beta=1$. The instability problems of torque decentering nature and torque curves intersection appear in both cases. Because of the flow torque resulting when $\beta=0.5$ is less than that resulting when $\beta=1$, the required dimensionless stiffness to avoid instability is less in the earlier case than in the latter one. This implies less driving torque and/or higher allowable supply pressure and output power. Figure 8 shows the dimensionless output power and the efficiency when $\beta=1$ while Fig. 9 shows them when $\beta=0.5$. Comparing between

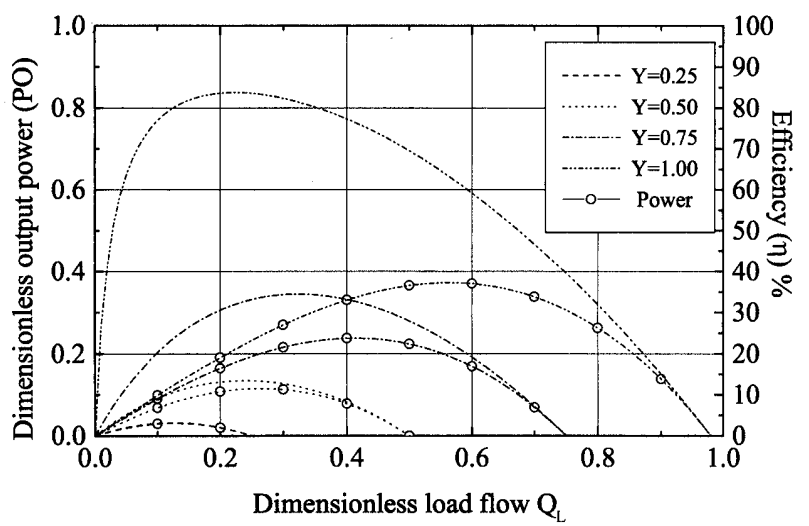

Fig. 8 Variation of efficiency and dimensionless output power with dimensionless load flow for various dimensionless input displacement under constant supply pressure operation when $\beta=1$

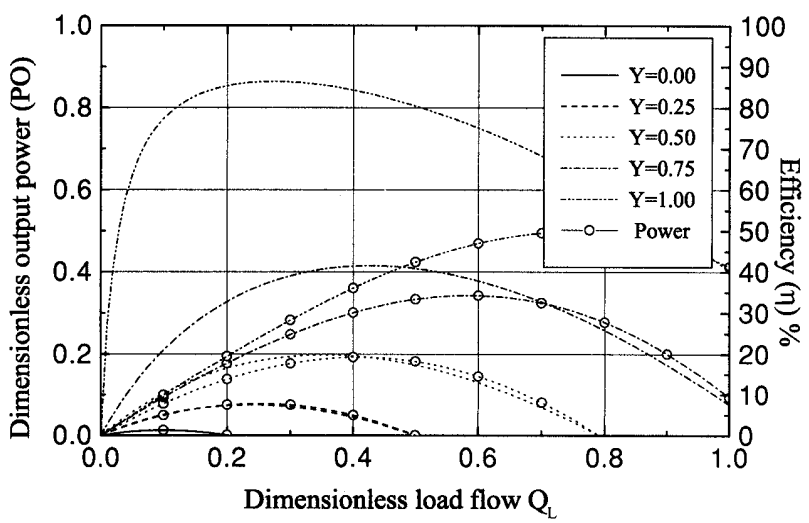

Fig. 9 Variation of efficiency and dimensionless output power with dimensionless load flow for various dimensionless input displacement under constant supply pressure operation when $\beta=0.5$ the values at both cases, it can be seen that the dimensionless output power and the efficiency are higher in case of $\beta=0.5$ than in case of $\beta=1$.

\section{Valve Static Characteristics under Constant Supply Flow Operation}

In this case the supply flow is constant. The governing equations can be reduced to a dimensionless form using the following reference quantities;

$i$-the reference flow is the supply flow

$$
q_{r}=q_{s}
$$

$i i$-the reference pressure is the null supply pressure

$$
p_{r}=0.5\left[\frac{q_{r}}{b}\right]^{2}
$$

$i i i$-the reference input displacement is the null gap $y_{r}=y_{n}$

$i v$-the reference torque is taken as

$$
m_{r}=\frac{\rho . B}{2 A} q_{r}^{2}
$$

$v$-the dimensionless stiffness is

$$
K=\frac{2 k \cdot C \cdot A \cdot y_{n}}{\rho \cdot B \cdot q_{r}^{2}}
$$

Uppercase letters will be used to express the quantities in dimensionless form. The cavitation limits when $\beta=1$ can be shown to be;
a. For $P_{1}=P_{4}=0$

$$
Q_{L}=1
$$
b. For $P_{2}=P_{3}=0$

$$
Q_{L}=-1
$$

Regarding the torque acting on the pintle due to the flow forces only, i.e. at $K=0$. It can be shown that when $\beta=1$

$$
M_{f}=2 Q_{L}
$$

i.e. the flow torque is only dependent on the load flow. Elgamil $^{(3)}$ showed that when $\beta=0.5$, the relation is not one perfect line for all input displacements, and that below the dimensionless load flow $Q_{L}=0.5$, the flow torque when $\beta=0.5$ is only $75 \%$ of the flow torque when $\beta=1$, and the reduction increases above this dimensionless load flow. Figure 10 shows variation of dimensionless load pressure with dimensionless load flow for various dimensionless input displacements when $\beta=1$. The figure shows that the $P_{L}-Q_{L}$ relation is not linear over a wide range like that of the performance under constant supply pressure conditions. Besides, it shows the effect of the error in one gap length on the $P_{L}-Q_{L}$ relation where case no. 1 and case no. 2 are as described in section 3 . It is seen that the differences created by the errors under both constant supply pressure and constant supply flow operation are in the same sense. However the variations under constant supply flow operation are seen to be smaller than those obtained under constant supply pressure operation. These differences are seen to 
decrease with the increase of the input displacement value. While, the maximum supply flow under constant supply pressure operation conditions is within 1.3 times the null flow rate (Fig. 5), the maximum supply pressure under constant supply flow operation condition is high. Figure 11 shows that the supply pressure may reach 8 times the null supply pressure at $75 \%$ of the maximum input displacement and blocked load conditions. This means that the system may reach the preset maximum pressure at relatively small input displacement. Figure 12 shows the variation of the dimensionless load pressure with the dimensionless load flow for various input displacement and two different load area ratios. Except for the early appearance of cavitation when $\beta=0.5$, there is no big difference between the two cases. Figure 13 shows the dimensionless output power and the efficiency when $\beta=1$ while Fig. 14 shows them when $\beta$ $=0.5$. Comparing the two figures, it is seen that the efficiency and the dimensionless output power are

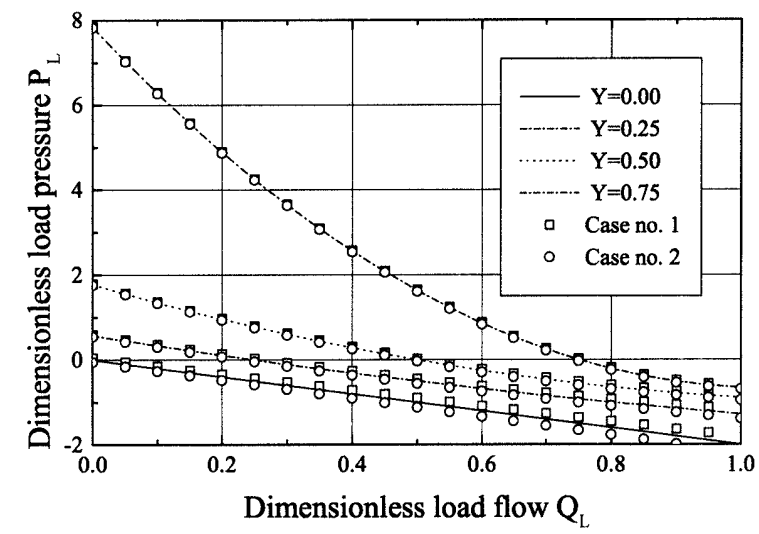

Fig. 10 Variation of dimensionless load pressure with dimensionless load flow for various dimensionless input displacement under constant supply flow operation and errors effect when $\beta=1$

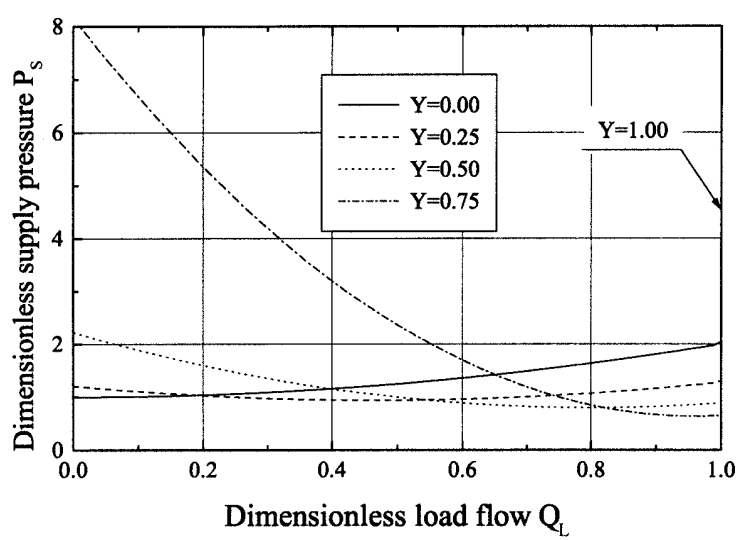

Fig. 11 Variation of dimensionless supply pressure with dimensionless load flow for various dimensionless input displacements under constant supply flow operation when $\beta=1$ improved when $\beta=0.5$. Generally speaking, the valve operation under constant supply flow operation shows to render higher output power and efficiency compared with the constant supply pressure operation. However, this is accompanied with increasing the

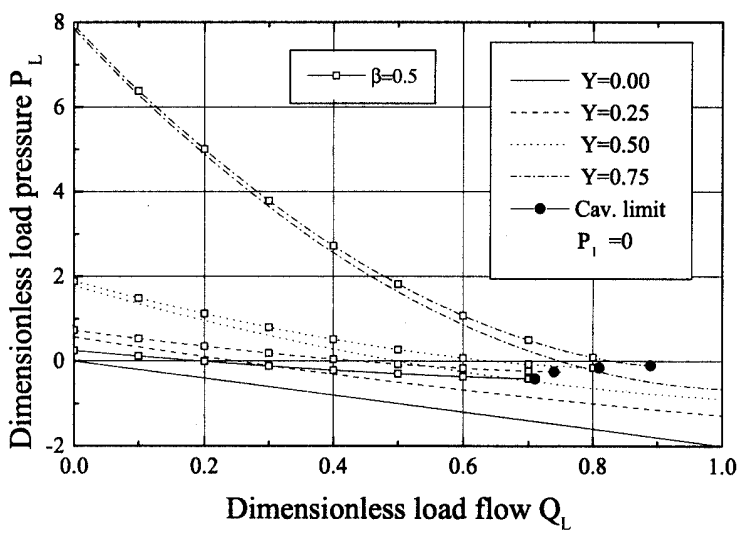

Fig. 12 Variation of dimensionless load pressure with dimensionless load flow for various dimensionless input displacement under constant supply flow operation when $\beta=1$ and $\beta=0.5$

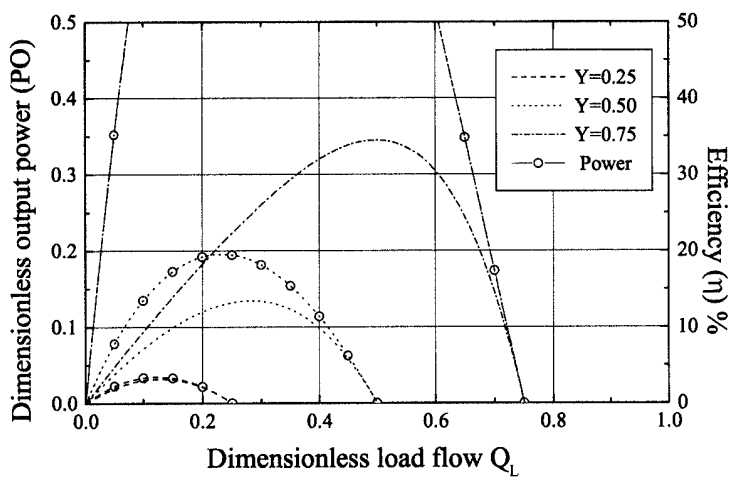

Fig. 13 Variation of efficiency and dimensionless output power with dimensionless load flow for various dimensionless input displacement under constant supply flow operation when $\beta=1$

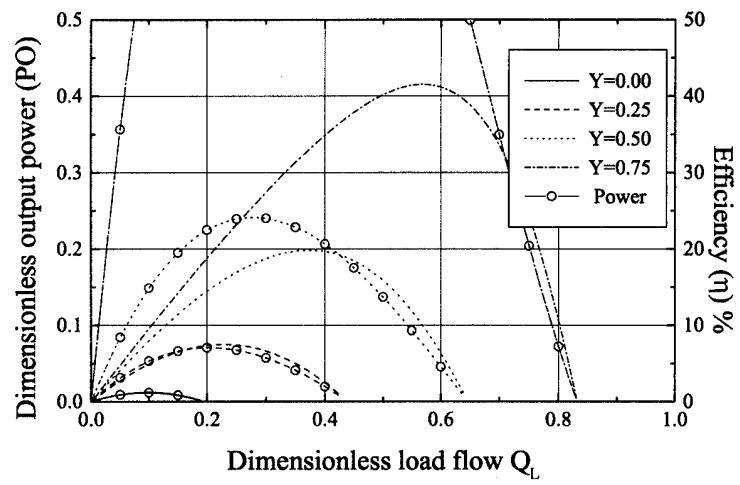

Fig. 14 Variation of efficiency and dimensionless output power with dimensionless load flow for various dimensionless input displacement under constant supply flow operation when $\beta=0.5$ 
supply pressure to large values.

\section{Conclusion}

The torque acting on the pintle due to flow forces is found to be of a decentering nature, and may result in instability problems. These problems can be overcome through the proper choice of the parameters of a centering spring. The minimum value of the spring stiffness, corresponding to a certain supply pressure to avoid instability, can be deduced in view of the presented relations. Errors in valve control gaps showed to have little effect on the valve flow gain and pressure coefficient. Equal errors of control gapes may duplicate or cancel their effects, depending on the error sign, on the performance. When a four-nozzle pintle type hydraulic servovalve is used for driving an unsymmetrical actuator, the driven unit would be liable to move when no input signal is applied to the valve. Therefore, the centering spring have to be adjusted in a position depend on the actuator area ratio and not at the midway between the nozzles. The flow torque applied to the pintle is less than the flow torque if a symmetrical actuator is used, therefore the centering spring can be with less stiffness and/or the supply pressure and the output power can increase. The valve flow gain, the flow-pressure coefficient, the output power and the efficiency increase when an unsymmetrical actuator is used. Systems with sym- metrical actuators have the minimum damping ratio.

\section{References}

(1) Williams, L.J., High Performance Single Stage Servovalve, SAE Aerospace Fluid Power Systems and Equipment Conference, Los Angles, California, May 18-20, 1965, (Moog Technical Bulletin 106).

(2) Kassem, S.A., and Arafa, H.A., Design Aspects of Four-Nozzle Hydraulic Servovalves, International Journal of Machine Tools Manufact., Vol. 27, No. 4 (1987), p. 457-468.

(3) Elgamil, M.A., Investigation of Performance of Single Stage Hydraulic Servovalves with Four Control Gaps M. Sc. Thesis, Faculty of engineering, Cairo University, (1991).

(4) Kassem, S.A. and Arafa, H.A., Static and Dynamic Characteristics of Four-Nozzle Flapper Valves, Proceeding of 10th Conference on Fluid Mechanism, Brno, Czechoslovakia, 2-4 November 1982, p. 172-195.

(5) Bahr, M.K., Theoretical and Experimental Investigation of Performance of Four-Nozzle Hydraulic Servovalves, M. Sc. Thesis, Faculty of engineering, Cairo University, (1988).

(6) Osman, T.A., Design and Performance of FourNozzle Valves M. Sc. Thesis, Faculty of engineering, Cairo University, (1983).

( 7 ) Merritt, H.E., Hydraulic Control Systems, (1967), John Wiley \& Sons Inc. 